\title{
Smart Surveillance Security Systems 4s for Detection using SIFT and SURF in Image Processing
}

\author{
Aghila Rajagopal, R. T. Subhalakshmi, Arunachalam, D. Deepika, N. Balaji
}

\begin{abstract}
Surveillance video is used for security purpose in our daily life in various places. It is used to observe the unusual activity that is taking place around us. Today in most of the shop owners have CCTV cameras to record, the uncertain activities and even it is used in houses in remote places. A system must be smart enough to detect. This paper uses SIFT and SURF algorithm for detection. Image registration is a development in which more than two images from various imaging equipment are reserved at various angles and at various times from the identical prospect and geometrically aligned for further exploration. Data may be from different sensors, CCTV taken at different times, depths, or perspective. Feature-DetectorDescriptor plays a vital role in feature matching application for selection of feature; this paper presents a comparative analysis of SIFT, SURF, algorithms. Experiments have been conducted on a wide range of images taken from datasets. A quantitative comparison is presented. This paper gives an useful ideas for making important decisions and it also helps in providing a smart security system.
\end{abstract}

Keywords-SIFT; SURF; image registration; nearest neighbor distance ratio; feature matching; scale invariance; rotation invariance; affine invariance; image matching; feature detection;

\section{INTRODUCTION}

Image registration applications are Real Time Alerts which includes user defined alerts, Generic Alerts, Classic Specific Alerts and Behavioral Alerts for Video Processing. Developing an optimized method that suits for all users as there are wide ranges of application due to images geometric radiometric distortions and noise disturbance, data characteristic and accuracy threshold level are still in process.

Revised Manuscript Received on February 05, 2020.

* Correspondence Author

Dr. R. Aghila*, Department of Information Technology, Sethu Institute of Technology, Kariapatti. Tamilnadu, India.

Email: aghila25481@gmail.com

R. T. Subhalakshmi, Department of Information Technology, Sethu Institute of Technology, Kariapatti. Tamilnadu, India.

Email: subhalakshmirt@gmail.com

Dr. M. Arunachalam, Professor, K.L.N .College of Information Technology, Pottapalaym,Sivagangai (Dt), Madurai, Tamilnadu, India. Email: er.arun80@gmail.com

D. Deepika, Department of Computer Science and Technology, K.L.N

College of Information Technology, Pottapalaym, Sivagangai (Dt), Madurai. Tamilnadu, India. Email: deepika5334@gmail.com

Dr. N. Balaji, Head, Department of Computer Science and Engineering in KPR Institute of Engineeirng and Technology, Coimabtore, India. Email: balajin.ithod@gmail.com

(C) The Authors. Published by Blue Eyes Intelligence Engineering and Sciences Publication (BEIESP). This is an open access article under the CC BY-NC-ND license (http://creativecommons.org/licenses/by-nc-nd/4.0/)

The significance of image registration techniques develops with developing number of different types of data acquisition devices and their increasing availability. Objects needed for image registration can be aligned that can be simply translated and rotated to one another.

\section{LITERATURE SURVEY}

\section{A. Scale Invariant Feature Transform (SIFT):}

The most renowned feature-detection-description algorithm, Scale Invariant Feature Transform (SIFT) was introduced by D. G. Lowe in 2004 [13]. SIFT detector is based on Difference-of-Gaussians (DoG) operator, an approximation of Laplacian-of-Gaussian (LoG). DoG is preferred because of low computational complexity and it does not need partial derivative computation. It also obtains local extrema of images with a difference of the Gaussian.

SIFT algorithm can be divided into four steps:

- DoG Generation

- Keypoint Detection

- Orientation assignment

- Feature descriptor generation.

It is a technique used for detecting prominent, stable feature points in an image. It also responsible for a set of -featuresll describes a small image region around the point. These features are invariant to rotation and scale. The applications need to sense the unwavering points in two or more image and determine the communications between them. In order to determine the communications correctly, we need some features portraying a significant point. These features obligation does not change with the Object position or pose, Scale, Enlightenment and the Minor image artifacts.

We can also continue with the nearby matching patches around the feature points, but they will transformed to themselves, if there is a change in pose or illumination and they may lead to false matches. It remains invariant to changes in scale or rotation.

DoG images are produced by image convolving with Gaussians at each octave of the scale space in the Gaussian Pyramid. Gaussian image is based on the number of octaves. It is down sampled in every iterations.Key point generation involves key point localization. Each pixel in the DoG image is matched to its neighboring pixels. Key points can be local maximum or local minimum. The final sets of key points exclude low contrast points. 
Key point orientation is the local image gradient histogram in the neighborhood of the key point. Dominant orientations are selected as the peaks in the histogram. For each key point, feature descriptors are computed.

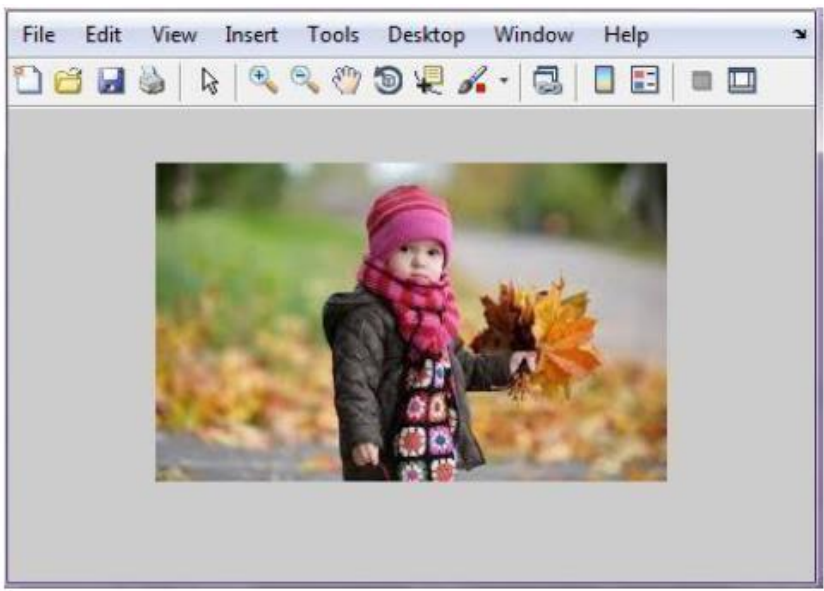

Original Image

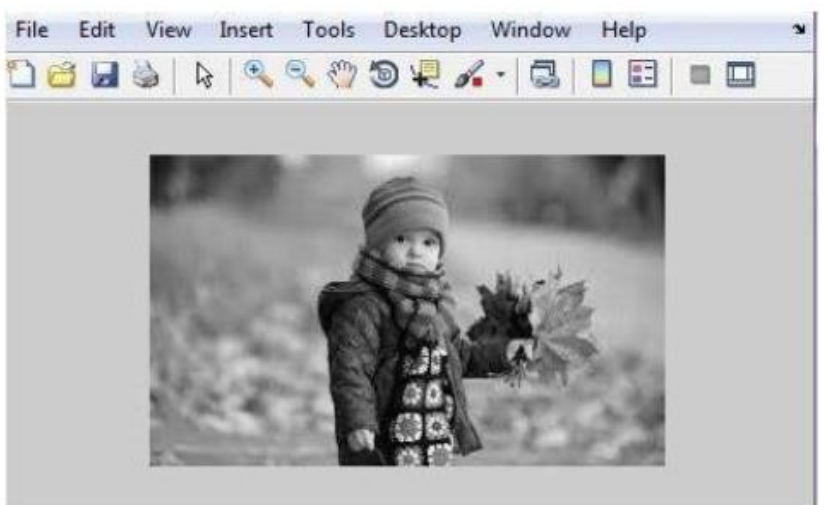

Gray scale Image

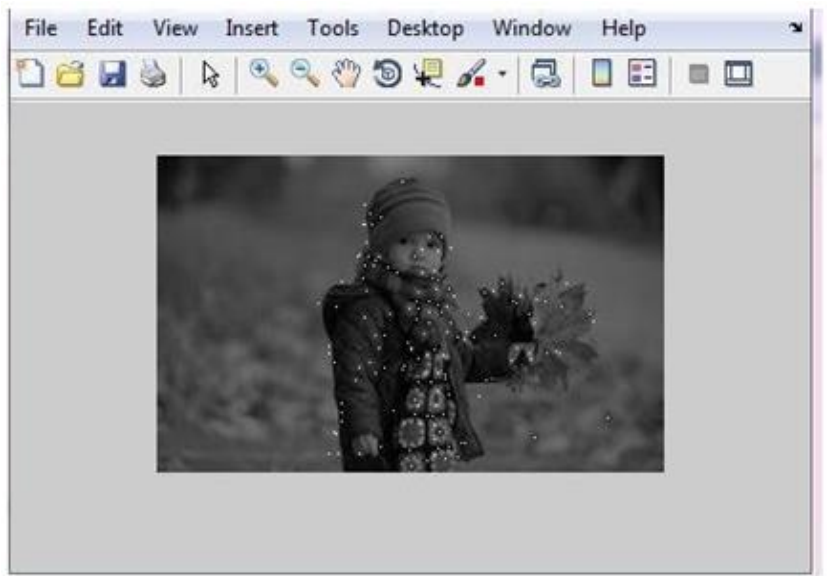

SIFT Feature Extracted

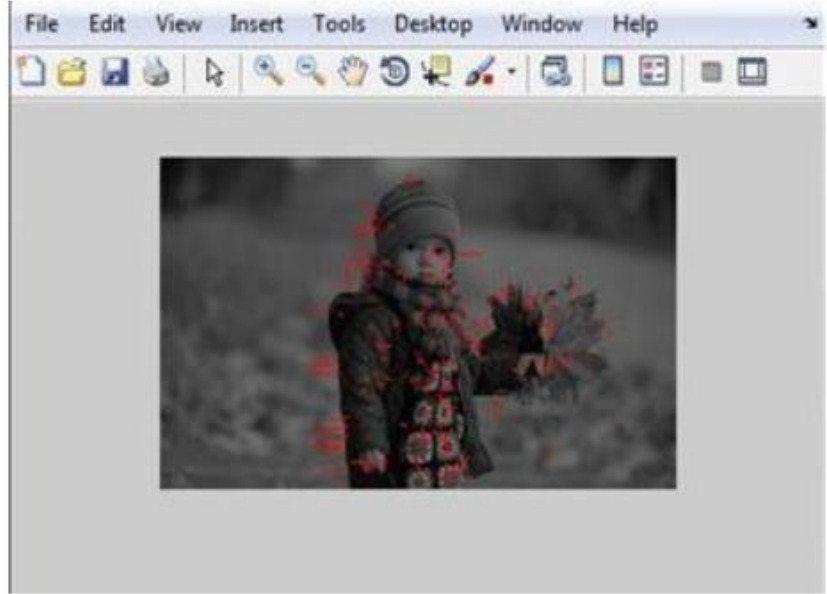

\section{SIFT Feature Extraction with Gaussian Value}

B. Speeded Up Robust Features (SURF):

It is a upgraded technique of SIFT. It uses approximate Gaussian second derivative mask to an image at varies scales. SURF is a fast technique, because of the procedure of an integral image where the assessment of a pixel $(x, y)$ is the amount of all ethics in the rectangle defined by the source and $(\mathrm{x}, \mathrm{y})$. The foundation image can be found as the outcome of 4 operations. It facilitates a very little computing time, as it allows a rectangular mask of any size to be applied. To detect features, we assemble the Hessian matrix. The SURF descriptor is designed to be scale invariant and rotationally invariant. After generating feature descriptor, the dominant orientations of the area are rotated to the dominant orientation of the feature points. For analysis we used the functions SURF.detect(), SURF.compute() for finding keypoints and descriptors. 1199 keypoints is too much to show in a picture. We reduce it to some 50 to draw it on an image. While matching, we may need all those features, but not now. So we increase the Hessian Threshold. If we apply U-SURF, we won't find the orientation. All the orientations are shown in same direction. It is faster than the SIFT.

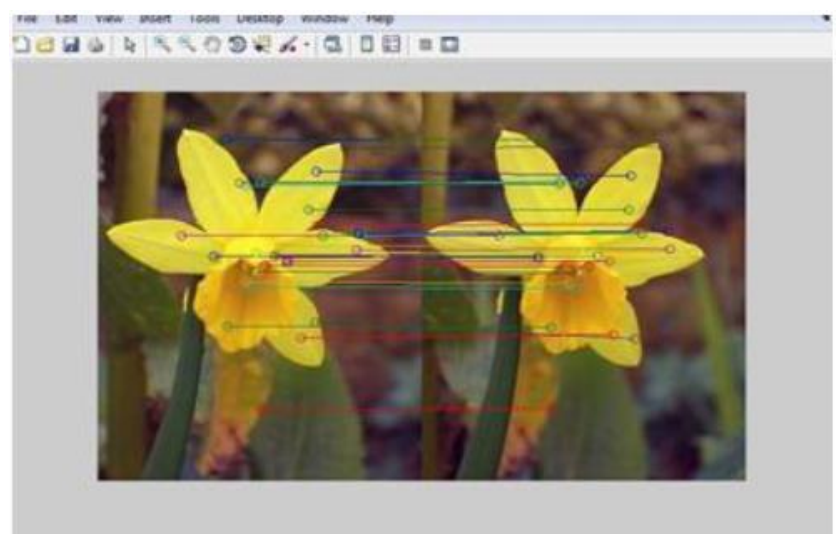

Output of the wrapped figure

\section{EXPERIMENTS \& RESULTS}

To verify the effectiveness of the two algorithm images are taken as the experimental data. Features are detected for images using SIFT and SURF algorithm. In SIFT number of scale space intervals are given as 3. Gaussian kernels also calculated.

\section{Published By:}

Blue Eyes Intelligence Engineering \& Sciences Publication 
For calculating accurate local key point set the threshold value as

0.03 . The patch size for dominant orientation calculation is 9 and the patch size for feature transformation is 16 . With the scale space corner index the features are extracted and the output is displayed for SIFT. SURF detects the points by a vector which is robust against rotation, Scaling and noise. Hessian response threshold is given as 0.0002 . With the help of many sub functions the SURF extract the features from the given image and the output are mentioned above. The investigational outcomes provide amusing figures and different original insights that are treasured for producing critical assessments in Smart Surveillance Security System. The Table 1 shows how the feature extractions of two algorithms are analyzed with some parameters.

Table 1: Comparison between SURF and SIFT

\begin{tabular}{|c|l|c|c|}
\hline $\begin{array}{c}\text { S.N } \\
\mathbf{0}\end{array}$ & \multicolumn{1}{|c|}{$\begin{array}{c}\text { Para } \\
\text { mete } \\
\text { rs }\end{array}$} & SIFT & SURF \\
\hline 1 & Accuracy & High & $\begin{array}{c}\text { Modera } \\
\text { te }\end{array}$ \\
\hline 2 & Detect High $\begin{array}{c}\text { Quality Of } \\
\text { Features }\end{array}$ & $\begin{array}{c}\text { Modera } \\
\text { te }\end{array}$ & High \\
\hline 3 & Computational Efficiency & $\begin{array}{c}\text { Modera } \\
\text { te }\end{array}$ & High \\
\hline 4 & $\begin{array}{l}\text { Matching Per Feature-Point } \\
\text { for effective matching }\end{array}$ & $\begin{array}{c}\text { Modera } \\
\text { te }\end{array}$ & High \\
\hline
\end{tabular}

\section{CONCLUSION}

This paper provides a complete assessment of SIFT and SURF based on feature-detector-descriptors. SIFT, and SURF are conventional as the most balanced invariant feature detectors established on the foundation of repeatability, that have persisted comprehensive extent scale dissimilarities. SIFT have greater accuracy as associated with SURF for image rotations. The comprehensive accurateness of SIFT is establish to be maximum than SURF for all categories of geometric conversions and SIFT is determined as the most truthful algorithm compared to SURF. The capability to distinguish great quality of structures is SURF than SIFT. The computational efficiency of feature-detection- description per feature-point is better in SURF than in SIFT. But the order of proficient feature-matching per feature-point is better in SURF as mentioned in table. Further we implement this technique in video registration.

\section{REFERENCES}

1. P M Panchal, S R Panchal, S K Sha, -A Comparison of SIFT and SURFI, International Journal of Innovative Research in Computer and Communication Engineering, Vol. 1, Issue 2, April 2013

2. Bay, H., Tuytelaars, T., Van Gool, L.: SURF: Speeded Up Robust Features. In: Leonardis, A., Bischof, H., Pinz, A. (eds.) ECCV 2006. LNCS, vol. 3951, pp. 404-417. Springer, Heidelberg (2006).

3. 1Utsav Shah, 2Darshana Mistry, 3Yatin Patel "Survey of Feature Points Detection and Matching using SURF, SIFT and PCA-SIFT" Journal of Emerging Technologies and Innovative Research ISSN2349-5162 Volume 1 Issue 1

4. Sajid Saleem1, Abdul Bais2, and Robert Sablatnig3 -A Performance Evaluation of SIFT and SURF for Multispectral Image Matching\| International Conference Image Analysis and Recognition 2012

5. Prashant Aglave1 V.S. Kolkure2,\| Comprehensive Survey on Image Feature Extraction Techniques\| IJSRD - International Journal for Scientific Research \& Development| Vol. 2, Issue 11, 2015 | ISSN (online): 2321-0613.

6. Shaharyar Ahmed Khan Tareen, Zahra Saleem -A Comparative Analysis of SIFT, SURF, KAZE, AKAZE, ORB, and BRISK॥, 2018 International Conference on Computing, Mathematics and Engineering Technologies -iCoMET2018,IEEE

\section{AUTHORS PROFILE}

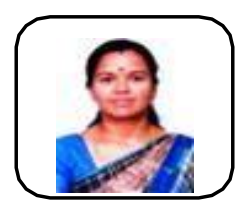

Dr. R. Aghila, Associate Professor of Department of Information Technology, Sethu Institute of Technology, has more than 15 years of teaching experience. She received her Ph.D degree from Anna University, Chennai in 2015. Her research area includes Distributed Computing, Software Engineering. She has published many papers in National and International journals. She is a reviewer in various national, international Conferences and Journals. She holds the membership in many Professional bodies like CSI, IAENG, IEDRC, IACSIT.

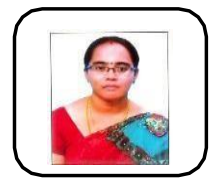

R. T. Subhalakshmi, received her B.Tech.Degree from Odaiyappa College of Engineering and Technology, India in 2011 and M.Tech from K.L.N. college of Technology, India in 2013. Since 2017 she has been a research Scholar, pursuing her Ph.D in Anna University, Chennai, India. She has 10 Journals/Conference Publications. Currently she is working as Assistant Professor, Department of Information Technology, Sethu Institute of Technology. Her research interest includes Image Processing, Steganalysis, Deep learning. She is a Life Member of Indian Society for Technical Education.

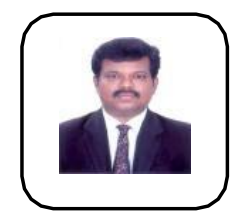

Dr. M. Arunachalam, holds B.E. Degree in Computer Science and Engineering from Madurai Kamaraj University in 2001 and M.E. Degree in Computer Science and Engineering from Anna University in 2004. He was awarded Doctorate Degree in Optical Networks from Anna University, Chennai in 2013 India. He has published 40 papers in Journals and conferences at National and International levels till date. $\mathrm{He}$ has received and associated in receiving research grants from recognized government funding agencies. He has acted as Resource Person and Session Chair in many reputed international conferences worldwide. His research interests are Communication Networks and Internet of Things.

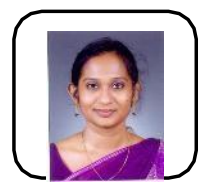

D. Deepika, received her B. E. Degree from SACS Engineering College, India in 2011 and M.E from Kumaraguru college of Technology, India in 2013. Since 2018 she has been a research Scholar, pursuing her Ph.D in Anna University, Chennai, India. Currently she is working as Assistant Professor, Department of Computer Science and Engineering, K.L.N. College of Information Technology. Her research interest includes Artificial intelligence, Machine Learning.

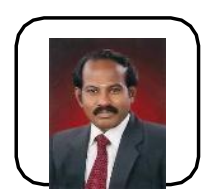

Dr. N. Balaji, currently serves as Head of the Department of Computer Science and Engineering in KPR Institute of Engineeirng and Technology, Coimabtore, India. He did his Bachelors in Electronics and Communication Engineering from

Madurai Kamaraj University, India. M.E. Degree Specializing in Computer Science and Engineering from Manonmanium Sundaranar University, India, Doctorate Degree in Computer Science from Anna Universiy, Chennai, India. He served as Faculty with Thiagarajar College of Engineering, Madurai and also acted as Head for Information Technology Department. He served for K.L.N.Group of Insitutions for more than 15 years at various capacities including Principal of K.L.N.College of Information Technology, Sivagangai. He also served in P.S.N.A. College of Engineering and Technology, Dindigul. He has

24 years of Teaching Experience. He has 26 Journals/Conference Publications and has authored one Book. Five candidates had completed their $\mathrm{PhD}$ under his able guidance. He is the member of Sub Syllabus Committee of Anna University, Chennai. He is a Life Member of Indian Society for Technical Education and is a Fellow of Institution of Engineers (FIE) India. 